\title{
Inteligencias múltiples y el aprendizaje de tecnologías en universitarios peruanos
}

\section{Multiple intelligences and technology learning in Peruvian university students}

\author{
Edith Mariela Quispe Sanabria \\ quispeedith627@gmail.com \\ https://orcid.org/0000-0003-1875-0048 \\ Lisette Paola Campos Carpena \\ lishi1905@gmail.com \\ https://orcid.org/0000-0003-2747-9456 \\ Lizet Doriela Mantari Mincami \\ d.Imantari@upla.edu.pe \\ https://orcid.org/0000-0003-4859-9684 \\ Universidad Peruana Los Andes (Perú)
}

Recibido: $23 / 01 / 21$

Aceptado: 10/05/21

\section{Resumen}

El propósito del trabajo es establecer la relación existente entre las Inteligencias Múltiples (IM) y el aprendizaje de Tecnologías de Información y Comunicación (TIC) en estudiantes universitarios peruanos. La investigación es de tipo aplicada, de diseño correlacional; la población es de 471 estudiantes universitarios peruanos. Los resultados demuestran que con $31.70 \%$ la mayoría de los estudiantes presentan un nivel alto en la variable IM; asimismo, el $54 \%$ de los estudiantes muestran un nivel medio en la variable aprendizaje de TIC. Como conclusión se tiene que existe una relación directa entre las IM y el aprendizaje de TIC.

Palabras clave: Inteligencia emocional; Tecnologías de la Comunicación e Información; TIC; Aprendizaje; Test; Educación.

\section{Abstract}

The purpose of the work is to establish the relationship between Multiple Intelligences (IM) and the learning of Information and Communication Technologies (ICT) in Peruvian university students. The research is of an applied type, of correlational design; the population is 471 Peruvian university students. The results show that with $31.70 \%$ most of the students present a high level in the MI variable; likewise, $54 \%$ of the students show

42 El aprendizaje a través de la ciencia ficción: El audiovisual Guardians of the heritage. - Eduweb, 2021, 
๔eduweb

Revista de Tecnología de Información y Comunicación en Educación • Volumen 15, Nㅜ 2. Mayo-agosto 2021

an average level in the ICT learning variable. As a conclusion, there is a direct relationship between IM and ICT learning.

Keywords: Emotional intelligence, Information and Communication Technologies, ICT, Learning, Test, Education.

\section{Introducción}

Bien es sabido que la teoría de las inteligencias múltiples (IM), ha provocado diversas discusiones desde su origen y son muchos los profesionales de la psicología que mantienen la postura de la existencia de la inteligencia única en los seres humanos, promoviendo ésta con los test de coeficiente intelectual $(\mathrm{Cl})$. Además, de acuerdo con Gardner (2015), muchos docentes parten de la premisa de que, si el estudiante presta atención a las diversas formas de pensamiento, de aprendizaje y evaluación, no logran progresar en los contenidos cursados.

La inteligencia, de acuerdo con Gardner (2015), constituye una cualidad presente en las personas en diversos niveles. Es el elemento fundamental para resolver problemas. Dicha cualidad puede evaluarse de manera confiable a través de test estandarizados respondidos con papel y lápiz, el cual puede predecir el futuro de los estudiantes en su formación escolar.

Según Bretell citado por Matheus (2011) argumenta que "el funcionamiento de cada inteligencia tiene que ver, por un lado, con su localización en el cerebro, y por el otro, con el desarrollo histórico de la misma" (p. 25). Es por ello que se puede aseverar que todas las personas nacen con la oportunidad de potencializar las ocho inteligencias, a menos que tenga dificultades o daños en el cerebro.

En ese sentido, surge la interrogante del porqué muchos individuos logran potenciar distintas habilidades, mientras que otras se les dificulta, tal como lo refiere Matheus (2011), que en la teoría de Gardner se ha expresado que esta tiene que ver más en los niveles de estimulación que se recibe y la motivación que se proporciona, generando en el individuo estímulos que afectarán o lograrán maximizar las ganas de aprender. Es necesario que se evite utilizar frases descalificadoras a los chicos para que no se estanquen en distintas áreas, aunque ese no sea su talento, trabajo que corresponde tanto a los educadores como en la mayor parte de los padres y así reforzar las destrezas en las que más destaca el estudiante.

Es por ello que el propósito del estudio estuvo centrado en establecer la relación entre las IM y el aprendizaje de la asignatura de Tecnologías de la Información y Comunicación (TIC) de los estudiantes de una universidad peruana. Para ello se evaluaron cada una de las ocho dimensiones de las IM y por el aprendizaje de TIC se evaluaron las cuatro siguientes dimensiones: creación de páginas web, configuración de diseño de blogger, creación de entradas sway y procesamiento de exámenes auto corregibles utilizando 
google form. Por lo que la hipótesis del estudio se centró en que existe relación significativa entre las IM (y sus dimensiones) y el aprendizaje de TIC.

Se ha destacado que, "al pasar décadas anteriores las TIC han causado un efecto considerable en distintos aspectos de la sociedad" (UNESCO, 2019, párrafo 1). Ha sido tanto su influencia que hoy por hoy se habla mucho sobre una sociedad con mayor conocimiento y experiencia (Pérez et al., 2018); es así que se ha logrado ver cómo se ha transformado el proceso de formación, creando un modelo para los distintos entes de educación al incorporar las TIC, mejorando la experiencia de educación al ligarla con tareas que se hacen cotidianamente pero variando la forma de recibir la información, obteniendo resultados en los estudiantes, pues se ve la evolución en la obtención de capacidades y competencias en su desempeño (Islas, 2017).

De la misma forma, la Organización de Naciones Unidas (ONU), realiza esfuerzos en conjunto con la Organización de las Naciones Unidas para la Educación, la Ciencia y la Cultura (UNESCO), para potencializar las tecnologías y promover el progreso hacia el cuarto Objetivo del Desarrollo Sustentable, que reside en que todos los escolares tengan una educación de calidad (UNESCO, 2019). Asimismo, con el propósito de generar y cumplir con políticas efectivas vinculadas al empleo de las TIC en el campo educativo, de acuerdo con la UNESCO (2019b), la ONU trabaja para asegurar que las diversas naciones puedan migrar a dichos entornos en la educación.

Por su parte, García (2014), precisa que hay una vinculación entre las IM y los elementos educativos, como son las habilidades sociales, la inteligencia emocional, autoeficacia, entre otras; las cuales pueden incidir de forma evasiva en el desarrollo académico en las emociones estables, por otro lado, el sexo femenino se ha destacado con puntuaciones mayores en las áreas de lingüística, relación interpersonal y visión espacial. De la misma manera, el progreso educativo orientado a poner en práctica estrategias de enseñanza - aprendizaje contribuye a construir recursos que provocan aprendizajes significativos en los estudiantes (Argüello y Collazos, 2008). A estos elementos, se suman las ideas de Matute (2013), que argumenta que los entes académicos se encuentran a la vanguardia sobre integrar el internet en sus métodos de estudios, crean los docentes la motivación para planificar métodos de enseñar a los estudiantes de distintas formas al usar medios tecnológicos que impactará de forma profunda en ellos. Asimismo, Díaz (2009), refiere que las competencias que integran en referencia a las TIC que realizan los docentes en universidades se ubican en el nivel medio. Por su parte, Aquirre (2018), precisa que, en el mundo, son cada vez más los recursos para la implementación, gestión y uso de las TIC en la educación para asegurar la calidad en la enseñanza-aprendizaje. Por otra parte, Alarcón et al. (2014), precisaron que las TIC y el aprendizaje del inglés tienen una vinculación importante en los estudiantes de Idiomas de una provincia peruana.

En cuanto a la teoría que fundamenta la investigación, se encuentra la de las IM, ésta surgió a finales del siglo XX, creada por Howard Gardner en 1993, de la que emergió la forma de apreciar el significado de la inteligencia (Gardner, 2015). Entre las principales 
ideas planteadas en ésta es desestimar la noción de una única inteligencia, cuya génesis es genética y propone la presencia de diversas clases de inteligencia, identificando el aporte de la predisposición biológica, considerando de gran valor la contribución de la repercusión social en su desarrollo (Mesa, 2018).

De acuerdo con Moreno y Planells (2016), refieren que Gardner conceptualiza la inteligencia en los humanos "como el potencial de solucionar problemas o generar cosas identificables por uno o deferentes entornos culturales" (p. 201). Sin embargo, el más grande aporte de dicha teoría es el enfoque de la inteligencia hacia una ventana más amplia de la capacidad cognitiva, es por ello, las diversas inteligencias que supone la teoría de las IM; de la misma manera, la relevancia que tiene sobre esta, los factores biológicos y sociales. Adicionalmente, otro elemento fundamental en la teoría de las IM es la codificación, de acuerdo con Athanassopoulos y López (2017), argumentan que en la gran variedad de la inteligencia se utiliza un sistema de codificación que supone la creación de símbolos diferentes que ayudan a regular la información y a organizar, siendo un elemento cultural para almacenar todos los datos que se le suministra al individuo. En ese orden de ideas, Gardner en su teoría de las IM, considera diversas inteligencias como lo son: lingüística, lógico-matemática, espacial, musical, kinestésica, intrapersonal, interpersonal y naturalista; dando entender que dichas inteligencias se desarrollan en una persona en proporciones mayores o menores de acuerdo con sus experiencias en la que también inciden el proceso de enseñanza aprendizaje (Hidalgo et al., 2018).

Desde esta perspectiva, las IM intervienen como procesos educativos para así fomentar en los alumnos enseñanzas y aprendizajes de las diversas áreas, promoviendo la especificidad a través de labores enfocadas al desarrollo de cada una de los contextos de capacidad del estudiante, desde la niñez, facilitando a los maestros la aplicación de estrategias tendentes a la enseñanza multimodal para los discentes, permitiendo un aprendizaje integral (Calle et al., 2016).

Respecto a las TIC, estas han tenido un gran avance y evolución, empleándose en diversos casos como, por ejemplo, el educativo. Sobre estas, Gómez (1991), citado por Pérez (2017), precisa que se constituyen como "un conjunto de mecanismos, redes y soporte que unidos conforman un sistema de información conectado" (p. 257), por lo que forman un sistema apoyado en la informática, la tecnología y las telecomunicaciones. Por su parte, Cabero et al (2016), refieren la importancia de las TIC en el aprendizaje colaborativo, puesto que mejora el aprendizaje y el rendimiento académico en los estudiantes e incrementa la motivación para realizar las actividades en equipo.

Dichas tecnologías han tenido gran avance y crecimiento exponencial en los últimos años, por lo que Paucar et al. (2017), manifiestan que estas se han constituido en herramientas muy útiles en las actividades cotidianas, es por lo que constantemente se requiere la actualización sobre la manipulación y tratamiento de estas. En ese sentido, Acosta (2016), expresa que el impacto de las TIC ha trascendido a todos los contextos 
de la vida cotidiana, influyendo positivamente en vida cotidiana de las personas, independiente de la lengua, edad o sexo. Es por ello, que Montilla et al. (2018), expresan la relevancia de incorporar en el contexto académico las TIC, como es el de las geociencias; como recurso en las sesiones de clase y así poder fomentar y desarrollar los aprendizajes en los bachilleres y futuros docentes de un Instituto Pedagógico.

En ese orden de ideas, Chancusig et al. (2019), argumenta que "las TIC contribuyen a mejorar el intercambio por medio de las redes, lo social, cultural, técnico, profesional, debido a que pueden realizarse estudios a través de aulas virtuales y diversas plataformas" (p. 1343). Sin embargo, Aguilar (2012), expone que las TIC han llegado al ámbito educativo desde hace mucho tiempo y en algunos contextos se han aplicado sin ser exitosas; no obstante, en la actualidad la discusión no gravita entre su vinculación o no al proceso educativo, sino en que necesitan novedosas formas de observar e impulsar el aprendizaje.

Ahora bien, desde el punto de vista de Llorente y Cabero (2005) citado por Arras et al. (2011), en el presente la alfabetización tecnológica se tiene como base fundamental en el desarrollo formativo de los universitarios, todo lo cual deben tener dominio de las formas de interactuar en los nuevos formatos digitales y redes de comunicación. Por su parte, para Hermosa (2015), el empleo de las TIC en las instituciones educativas aún es restringido por elementos como son los recursos, estímulos para la migración, los saberes adecuados, la motivación, las políticas educativas, entre otros factores. En el contexto de estudio de las TIC en el entorno educativo, Heredero y Garrido (2017), señalan que estas contribuyen a la integración en el aula de manera integral, estimulando el aprendizaje y flexibilizando las necesidades y capacidades individuales en los estudiantes.

\section{Metodología}

El presente estudio corresponde a un tipo de investigación básica, con nivel y diseño correlacional, mediante el cual se establece la relación entre las variables de estudio (Hernández y Mendoza, 2018); para este caso: inteligencias múltiples y aprendizaje de tecnologías de información y comunicación en estudiantes universitarios. Asimismo, se empleó el método descriptivo, debido a que busca describir el fenómeno (Hernández et al., 2014); es decir, identificar el progreso educativo de inteligencia múltiple de los bachilleres y demostrar la relación que se presenta entre las variables investigadas.

La investigación se ejecutó en el año 2019, en la ciudad de Huancayo - Perú, en la Universidad Peruana Los Andes (UPLA), Sede La Merced, Semestre Académico 2019II. La población del estudio estuvo constituida por 471 estudiantes cursantes en dicha Universidad. Por otro lado, la muestra se manejó por medio de un muestreo no probabilístico intencionado (Ñaupas et al., 2018), constituyéndose por 63 universitarios de la referida casa de estudios.

\footnotetext{
El aprendizaje a través de la ciencia ficción: El audiovisual Guardians of the heritage. - Eduweb, 2021,
mayo-agosto, v.15, n.2./42-54
} 
Para la recolecta de información se realizó empleando la técnica de la encuesta y como instrumento el test de inteligencias múltiples para medir la variable inteligencias múltiples (Gardner, 2015); para evaluar la variable aprendizaje de tecnologías de comunicación e información en estudiantes universitarios se empleó el fichaje y su instrumento las fichas bibliográficas, de resumen, de párrafo y la observación no estructurada (Arias, 2012), en la que se tomó como referencia la rúbrica de la primera unidad de la asignatura TIC II. Puesto que el test de inteligencias múltiples, es un instrumento estandarizado internacionalmente por su creador Howard Gardner, no fue necesario el proceso de validación del mismo mediante el juicio de expertos (Palella y Martins, 2012).

El estudio fue ejecutado en la UPLA, sede La Merced, semestre académico 2019-II; tomando como muestra estudiantes de un curso de la asignatura de TIC; en la que en seis sesiones de clase se empleó la rúbrica de esta para la primera unidad del curso, evaluando los criterios: manejo de la aplicación blogger de google, secuencia y estructura de blogger de google, elementos de construcción de un blog profesional y presentación de glogger de google, con puntajes según el alcance del logro de 0 a 5 . Asimismo, el test de $\mathrm{IM}$, en las que a través de 74 preguntas se evaluaron las ocho inteligencias: inteligencia matemática, inteligencia lingüística, inteligencia física-kinestésica, inteligencia musical, inteligencia espacial, inteligencia interpersonal, inteligencia intrapersonal e inteligencia naturalista; con puntaje para cada afirmación 1 = nunca; 2 = casi nunca; 3 = a veces; 4 = casi siempre; $5=$ siempre.

\section{Análisis y resultados}

Respecto los resultados descriptivos de la variable IM, los resultados obtenidos (Tabla 1) demuestran que en los estudiantes universitarios de la UPLA presentan un alto nivel de inteligencia matemática con $38.1 \%$, mientras que un $25.4 \%$ mostró un nivel medio, el $20.6 \%$ presentan un nivel bajo, mientras que un $15.9 \%$ mostró un nivel muy bajo en este tipo de inteligencia; por lo que se concluye que un gran número de estudiantes muestra un alto nivel en la inteligencia lógica matemática. En cuanto a la inteligencia lingüística, la mayoría de los estudiantes mostraron un bajo nivel en esta inteligencia, mientras que el $23.8 \%$ evidenciaron un nivel alto, asimismo, el $23.8 \%$ mostró un nivel muy bajo, finalmente un $20.6 \%$ presenta un nivel medio en este tipo de inteligencia, por lo que se afirma que un gran número de estudiantes tienen un bajo nivel en la inteligencia lingüística. En relación a la inteligencia física-kinestésica, se tiene que la mayoría de los estudiantes con un $30.2 \%$ presentan un nivel muy bajo, en tanto que un $27 \%$ muestra un alto nivel muy bajo en este tipo de inteligencia, solo el $25.4 \%$ presenta un alto nivel y un $17.5 \%$ presenta un nivel medio; por lo que se tiene que un gran número de estudiantes presenta un nivel bajo en la referida inteligencia. 
Edith Mariela Quispe Sanabria, Lisette Paola Campos Carpena, Lizet Doriela Mantari Mincami.

Tabla 1. Resultados absolutos y porcentuales de las inteligencias matemática, lingüística y física-kinestésica.

\begin{tabular}{lcccccc}
\hline \multirow{2}{*}{ Escala de medición } & \multicolumn{2}{c}{$\begin{array}{l}\text { Inteligencia } \\
\text { matemática }\end{array}$} & \multicolumn{2}{c}{$\begin{array}{c}\text { Inteligencia } \\
\text { lingüística }\end{array}$} & \multicolumn{2}{c}{$\begin{array}{c}\text { Inteligencia física - } \\
\text { kinestésica }\end{array}$} \\
\cline { 2 - 7 } & $\mathbf{F i}$ & $\%$ & $\mathbf{F i}$ & $\%$ & $\mathbf{F i}$ & $\%$ \\
\hline Alto & 24 & 38.1 & 15 & 23.8 & 16 & 25.4 \\
\hline Medio & 16 & 25.4 & 13 & 20.6 & 11 & 17.5 \\
\hline Bajo & 13 & 20.6 & 20 & 31.7 & 19 & 30.2 \\
\hline Muy bajo & 10 & 15.9 & 15 & 23.8 & 17 & 27 \\
\hline Total & 63 & 100 & 63 & 100 & 63 & 100 \\
\hline
\end{tabular}

Los resultados alcanzados (Tabla 2) evidencian que los estudiantes presentan un nivel bajo en la inteligencia musical con un $33.3 \%$, mientras que un nivel $25.4 \%$ mostraron un nivel alto, mientras que el $23.8 \%$ presentan un nivel muy bajo en este tipo de inteligencia, finalmente un $17.5 \%$ muestran un nivel intermedio; por lo que se resume que la mayoría de los estudiantes presentan un bajo nivel en la inteligencia musical. Por su parte, la inteligencia interpersonal en los estudiantes mostró un nivel alto con un $36.5 \%$, un nivel bajo con un $25.4 \%$, mientras que el $20.6 \%$ mostró un nivel muy bajo y un 17.5 presenta un nivel medio; concluyendo que la mayoría de los estudiantes presenta un nivel alto en la inteligencia interpersonal. Respecto a la inteligencia intrapersonal, se tiene que el $33.3 \%$ presente un alto nivel, el $25.4 \%$ muestra un nivel medio, un $22.2 \%$ presenta un nivel bajo, mientras que un $19 \%$ presenta un nivel muy bajo; concluyendo que la mayoría de los estudiantes tienen un alto nivel en la inteligencia intrapersonal.

Tabla 2. Resultados absolutos y porcentuales de las inteligencias espacial, interpersonal e intrapersonal.

\begin{tabular}{lcccccc}
\hline \multirow{2}{*}{$\begin{array}{c}\text { Escala de } \\
\text { medición }\end{array}$} & \multicolumn{2}{c}{$\begin{array}{c}\text { Inteligencia } \\
\text { musical }\end{array}$} & \multicolumn{2}{c}{$\begin{array}{c}\text { Inteligencia } \\
\text { interpersonal }\end{array}$} & \multicolumn{2}{c}{$\begin{array}{c}\text { Inteligencia } \\
\text { intrapersonal }\end{array}$} \\
\cline { 2 - 7 } & $\mathrm{Fi}$ & $\%$ & $\mathrm{Fi}$ & $\%$ & $\mathrm{Fi}$ & $\%$ \\
\hline Alto & 16 & 25.4 & 21 & 36.5 & 21 & 33.3 \\
\hline Medio & 11 & 17.5 & 11 & 17.5 & 16 & 25.4 \\
\hline Bajo & 21 & 33.3 & 16 & 25.4 & 14 & 22.2 \\
\hline Muy bajo & 15 & 23.8 & 13 & 20.6 & 12 & 19 \\
\hline Total & 63 & 100 & 63 & 100 & 63 & 100 \\
\hline
\end{tabular}

Los resultados obtenidos (Tabla 3 ) respecto a la inteligencia naturalista en los estudiantes, se tiene que un $38.1 \%$ de estos presentan un alto nivel, un $27 \%$ mostraron un nivel medio, mientras que el $19 \%$ mostraron un nivel bajo, finalmente el $15.9 \%$ presenta un nivel muy bajo en esta inteligencia; concluyendo que la mayoría de los estudiantes presenta un nivel alto en la inteligencia naturalista. Por su parte, los resultados de la inteligencia espacial los estudiantes muestran un alto nivel con un $34.9 \%$, mientras que un $23.8 \%$ es de nivel medio, en tanto que un $22.2 \%$ presentan un

\footnotetext{
48 El aprendizaje a través de la ciencia ficción: El audiovisual Guardians of the heritage. - Eduweb, 2021,
} 
nivel bajo y un $19 \%$ muestra un nivel muy bajo. Estos hallazgos permiten afirmar que la mayoría de los estudiantes tienen un alto nivel en esta inteligencia. En relación al resultado de la variable IM en los estudiantes, se tiene que estos muestran un alto nivel con un $31.7 \%$, mientras que un $23.8 \%$ muestra un nivel medio, $23.8 \%$ presenta un nivel muy bajo y un $20.6 \%$ tiene un nivel bajo, por lo que se resume que la mayoría de los estudiantes presenta un nivel alto en las IM.

Tabla 3. Resultados absolutos y porcentuales de las inteligencias naturalista, espacial y variable inteligencias múltiples.

\begin{tabular}{lcccccc}
\hline \multirow{2}{*}{ Escala de medición } & \multicolumn{2}{c}{$\begin{array}{l}\text { Inteligencia } \\
\text { naturalista }\end{array}$} & \multicolumn{2}{c}{$\begin{array}{c}\text { Inteligencia } \\
\text { espacial }\end{array}$} & \multicolumn{2}{c}{$\begin{array}{c}\text { Inteligencias } \\
\text { múltiples }\end{array}$} \\
\cline { 2 - 8 } & $\mathbf{F i}$ & $\%$ & $\mathbf{F i}$ & $\%$ & $\mathbf{F i}$ & $\%$ \\
\hline Alto & 24 & 38.1 & 22 & 34.9 & 20 & 31.7 \\
\hline Medio & 17 & 27 & 15 & 23.8 & 15 & 23.8 \\
\hline Bajo & 12 & 19 & 14 & 22.2 & 13 & 20.6 \\
\hline Muy bajo & 10 & 15.9 & 12 & 19 & 15 & 23.8 \\
\hline Total & 63 & 100 & 63 & 100 & 63 & 100 \\
\hline
\end{tabular}

En cuanto a los resultados descriptivos de la variable aprendizaje de TIC (Tabla 4), en la dimensión creación de páginas web, se tiene que el 33.3\% presenta un nivel bajo, mientras que un $28.6 \%$ presenta un nivel medio, el $19 \%$ presenta nivele alto y muy bajo respectivamente; por lo que se tiene que la mayoría de los estudiantes presentan un nivel bajo en dicha dimensión del aprendizaje de TIC. Para la dimensión configuración de diseños con blogger de la variable aprendizaje de TIC, se tiene que el $31.7 \%$ presenta un nivel bajo, mientras que el $25.4 \%$ mostró un nivel medio, el $22.2 \%$ presentó un nivel alto, finalmente un $20.6 \%$ presenta un nivel medio; por lo que se establece que la mayoría de los estudiantes presenta un nivel bajo en la referida dimensión. Adicionalmente, en la dimensión creación de entradas Sway, se tiene que el 34.9\% presenta un nivel alto, el $27 \%$ presenta un nivel medio, mientras que el $22.2 \%$ presenta un nivel bajo y un $15.9 \%$ presenta un nivel muy bajo; concluyendo que la mayoría de los estudiantes presenta un nivel alto en la referida dimensión.

Respecto a la dimensión procesamiento de exámenes auto corregibles con Google, se tiene que el $33.3 \%$ presenta un nivel bajo, el $25.4 \%$ muestra un nivel medio, mientras que el $22.2 \%$ presenta un nivel un nivel alto, finalmente el $19 \%$ presenta un nivel muy bajo, por lo que se considera que la mayoría de los estudiantes presenta un nivel bajo en esta dimensión. Resumiendo, se tiene que los estudiantes en el aprendizaje de TIC presentan un nivel medio en $54 \%$; mientras que el $28.6 \%$ presenta un nivel intermedio, el $11.1 \%$ presenta un nivel bajo, mientras que el $6.3 \%$ muestra un nivel muy bajo; por lo que se establece que de acuerdo con los resultados descriptivos los estudiantes tienen un nivel medio en el aprendizaje de TIC. 
Tabla 4. Resultados porcentuales de la variable aprendizaje de TIC.

\begin{tabular}{lcccccccccc}
\hline $\begin{array}{c}\text { Escala } \\
\text { de } \\
\text { medición }\end{array}$ & \multicolumn{2}{c}{$\begin{array}{c}\text { Creación } \\
\text { de } \\
\text { páginas } \\
\text { web }\end{array}$} & \multicolumn{2}{c}{$\begin{array}{c}\text { Configuración } \\
\text { de diseños } \\
\text { blogger }\end{array}$} & \multicolumn{2}{c}{$\begin{array}{c}\text { Creación } \\
\text { de } \\
\text { entradas } \\
\text { Sway }\end{array}$} & \multicolumn{2}{c}{$\begin{array}{c}\text { Proc. de exám. } \\
\text { auto } \\
\text { corregibles con } \\
\text { Google Form }\end{array}$} & \multicolumn{2}{c}{$\begin{array}{c}\text { Aprendizaje } \\
\text { de TIC }\end{array}$} \\
& $\mathbf{F i}$ & $\%$ & $\mathbf{F i}$ & $\%$ & $\mathbf{F i}$ & $\%$ & $\mathbf{F i}$ & $\%$ & $\mathbf{F i}$ & $\%$ \\
\hline Alto & 12 & 19 & 14 & 22.2 & 22 & 34.9 & 14 & 22.2 & 18 & 28.6 \\
\hline Medio & 18 & 28.6 & 16 & 25.4 & 17 & 27 & 16 & 25.4 & 37 & 54 \\
\hline Bajo & 21 & 33.3 & 20 & 31.7 & 14 & 22.2 & 21 & 33.3 & 7 & 11.1 \\
\hline $\begin{array}{l}\text { Muy } \\
\text { bajo }\end{array}$ & 12 & 19 & 13 & 20.6 & 10 & 15.9 & 12 & 19 & 4 & 6.3 \\
\hline Total & 63 & 100 & 63 & 100 & 63 & 100 & 63 & 100 & 63 & 100 \\
\hline
\end{tabular}

Como resumen de los resultados descriptivos porcentuales (Figura 1) en ambas variables, se tiene que los estudiantes en IM con un $31.70 \%$ presentan un nivel alto, el $23.80 \%$ presentan nivel medio y muy bajo respectivamente, mientras que un $20.60 \%$ presentan un nivel bajo; concluyendo que la mayoría de los estudiantes presentan un nivel alto en la referida variable. Respecto al aprendizaje de TIC, el 54\% presenta un nivel medio, el $26.60 \%$ presenta un nivel alto, mientras que el $11.10 \%$ presenta un nivel bajo, asimismo, el $6.30 \%$ presenta un nivel muy bajo en esta dimensión; concluyendo que la mayoría de los estudiantes tienen un nivel medio en la referida variable.

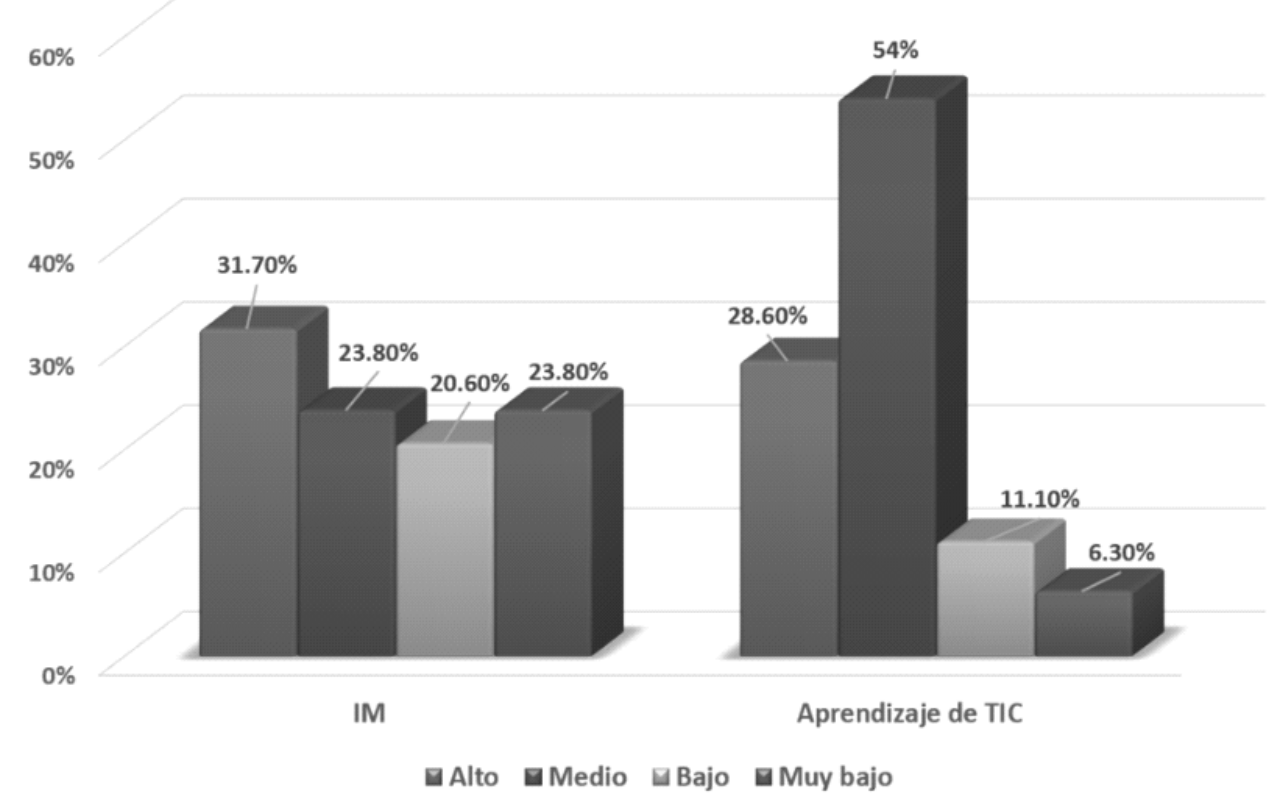

Figura 1. Resultados descriptivos porcentuales de las variables IM y aprendizaje de TIC 
Los resultados inferenciales (Tabla 5) se realizaron con el programa estadístico SPSS, en el que se calculó el coeficiente de correlación Rho de Spearman con un nivel de significancia del $0.05 \%$; el cual tiene como propósito evaluar la intensidad de una vinculación lineal entre dos o más variables (Rosas \& Zúñiga, 2010).

Tabla 5. Resultados inferenciales entre las variables IM y aprendizaje de TIC.

\begin{tabular}{|c|c|c|c|c|c|c|c|c|c|}
\hline $\begin{array}{l}\text { Corre- } \\
\text { lación }\end{array}$ & $\begin{array}{c}\text { Int. } \\
\text { Mat. } \\
y \\
\text { TIC }\end{array}$ & $\begin{array}{l}\text { Int. } \\
\text { Ling. } \\
\text { y TIC }\end{array}$ & $\begin{array}{c}\text { Int. } \\
\text { Física- } \\
\text { Kinest.y } \\
\text { TIC }\end{array}$ & $\begin{array}{c}\text { Int. } \\
\text { Musical } \\
\text { y TIC }\end{array}$ & $\begin{array}{c}\text { Int. } \\
\text { Espac. } \\
\text { y TIC }\end{array}$ & $\begin{array}{c}\text { Int. } \\
\text { Interper. } \\
\text { y TIC }\end{array}$ & $\begin{array}{c}\text { Int. } \\
\text { Intrap. } \\
\text { y TIC }\end{array}$ & $\begin{array}{l}\text { Int. } \\
\text { Natur. } \\
\text { y TIC }\end{array}$ & $\begin{array}{c}\text { IM y } \\
\text { aprend. } \\
\text { de TIC }\end{array}$ \\
\hline $\begin{array}{l}\text { Rho de } \\
\text { Spear- } \\
\text { man }\end{array}$ &, $768^{*}$ & ,823* &, $642^{*}$ & ,614* & ,831* & ,815* & ,758* & ,763* &, $841^{\star *}$ \\
\hline
\end{tabular}

Como se muestra en la Tabla 5, la correlación entre la dimensión inteligencia matemática y el aprendizaje de TIC de los estudiantes objeto de estudio, muestra un valor de 0.768 ; lo cual señala que entre estas existe una correlación positiva fuerte. Asimismo, entre la dimensión inteligencia lingüística y aprendizaje de TIC presenta un valor de 0.823 , lo que indica que existe una correlación positiva fuerte entre estas. Por otra parte, la dimensión inteligencia física - kinestésica y el aprendizaje de TIC en los estudiantes de la UPLA semestre 2017-II presenta un valor de 0.642; indicando que existe una correlación positiva media. De la misma forma, la dimensión inteligencia musical y el aprendizaje de TIC, muestra un valor de 0.614 ; lo que es indicativo de que existe una relación directa y la correlación entre estas es positiva media. Respecto a la dimensión inteligencia espacial y aprendizaje de TIC, presenta un valor de 0.831 ; lo que indica que existe una correlación positiva fuerte.

Respecto a la dimensión inteligencia interpersonal y aprendizaje de TIC, se obtuvo un valor de 0.815 indicando que existe una relación directa entre ambas y una correlación positiva alta. Por su parte, la dimensión inteligencia intrapersonal y el aprendizaje de TIC se obtuvo un valor de 0.758 , indicando una relación directa entre estas y una correlación positiva alta. Asimismo, la dimensión inteligencia naturalista y el aprendizaje de TIC, se alcanzó un valor de 0.763 , lo que señala que existe una correlación positiva fuerte entre estas. Finalmente, en los resultados inferenciales respecto a ambas variables, es decir, las IM y el aprendizaje de TIC, se obtuvo un valor de 0.841 ; indicando que existe relación entre ambas variables y una correlación positiva fuerte. Como cierre de los resultados, se tiene que se evidencia una fuerte relación entre las IM y el aprendizaje de TIC, demostrada con un coeficiente de correlación de Spearman de 0.841 , lo que señala que existe una correlación positiva fuerte; de manera similar, sucede con las dimensiones de las IM y el aprendizaje de TIC, en gran medida existe una relación significativa positiva fuerte, demostrando de este modo la hipótesis de la investigación. 


\section{Discusión y conclusiones}

De acuerdo con los resultados presentados anteriormente, están en concordancia con los de García (2014), en la que se estableció relación entre las variable IM y factores educativos como habilidades sociales, inteligencia emocional, autoeficacia; elementos que pueden repercutir de manera indirecta en el avance educativo y en un mejor equilibrio emocional. De la misma forma, se precisa que la vinculación entre las IM y el aprendizaje de TIC son directas a la clase de inteligencia que se produce en cada uno de los discentes. Por su parte, las investigaciones de Matute (2013), Arguello y Collazos (2008) y Aguirre (2018), orientadas a que en las instituciones conectadas a la internet sus maestros pueden reinventar los métodos y estrategias de enseñanzas; en ese sentido, es similar a los resultados de la presente investigación que apuntan a que el migrar de estrategias centradas en el docente impartiendo clases con el pizarrón y la tiza al uso de TIC puede tener un gran impacto en el proceso de enseñanza aprendizaje.

La investigación de Domínguez (2010), quien precisa que actividades relacionadas con las IM en las clases, generan una labor activa en el aula, incidiendo en el logro de las competencias; es por ello, que este estudio es similar, puesto que los resultados señalan un alcance en la rúbrica del aprendizaje TIC ubicado en nivel medio con un 54\% de los estudiantes. En la misma perspectiva, se presenta el trabajo de Alarcón et al. (2014), quienes establecieron que existe relación entre el aprendizaje de TIC y el idioma inglés en la muestra de su estudio, por lo que esta idea refuerza los hallazgos del presente estudio en el que con un coeficiente Rho de Spearman de 0.841 ; se establece que existe relación directa y correlación positiva fuerte entre las IM y el aprendizaje de TIC.

De acuerdo con las afirmaciones anteriores, se concluye que un estudiante de la UPLA que presente un nivel alto en IM presentará un alto nivel en el aprendizaje de TIC; asimismo, todo estudiante que presente un alto nivel en alguna de las dimensiones de las IM (matemática, lingüística, física-kinestésica, musical, espacial, interpersonal, intrapersonal y naturalista), presentará un alto nivel en aprendizaje de TIC. En ese sentido, se sugiere que en los procesos de orientación vocacional se aplique el test de IM, con el propósito de enfocar la aptitud de los estudiantes hacia las carreras que estén en concordancia con su perfil académico en función a la IM más desarrollada; tratando en gran medida de asegurar el alcance en sus logros y culminación exitosa. De la misma forma, se sugiere la implementación de estrategias, recursos y medios donde se involucren las TIC para potencializar el proceso de enseñanza aprendizaje en la UPLA.

\section{Referencias}

Acosta, L. (2016). La relación entre los estilos de aprendizaje y el uso de las tecnologías de información y comunicación en educación de personas adultas. Revista Electrónica Educare, 20(3), 199-216, https://bit.ly/3rLOkVA

Aguilar, M. (2012). Aprendizaje y Tecnologías de Información y Comunicación: Hacia nuevos escenarios educativos. Revista Latinoamericana de Ciencias Sociales, Niñez y Juventud, 10(2), 801-811, https://bit.ly/3o20wgL 
Arias, F. (2012). El Proyecto de investigación. Introducción a la Metodología Científica. (Sexta ed.). Caracas, Venezuela: Episteme.

Argüello, V.Y., y Collazos, L.A. (2008). Las inteligencias múltiples en el aula de clase. (Tesis de Maestría en Educación). Universidad Tecnologica de Pereira, PereiraColombia. https://bit.ly/2WGdrch

Aguirre, P. (2018). Las TIC en la gestión del proceso de enseñanza-aprendizaje en el área Comunicación Organizacional: licenciatura en Ciencias de la Comunicación. RIDE. Rev. Iberoam. Investig. Desarro. Educ, 8(16), 1-25. https://bit.ly/3mlkX0O

Arras, A., Torres, C., García, A. (2011). Competencias en Tecnologías de Información y Comunicación (TIC) de los estudiantes universitarios. Revista Latina de Comunicación Social, Vol. 66, 1-26. https://bit.ly/2WVLoFH

Athanassopoulos, N., López, V. (2017). Inteligencias múltiples y aprendizaje: Un enfoque comparativo en alumnos de conservatorio. ReiDoCrea, Vol. 6, 50-63. https://bit.ly/38OjpNI

Alarcón, D., Ramírez, M., Vílchez, M. (2014). Las tecnologías de información y comunicación (TIC) y su relación con el aprendizaje del idioma inglés de los estudiantes de la especialidad de inglés-francés, promoción 2011 de la Universidad Nacional de Educación Enrique Guzmán y Valle. Chosica, 2013. [Tesis de Maestría en Educación]. https://bit.ly/2WDHYr7

Cabero, J., Barroso, J., Llorente, M., Yanes, C. (2016). Redes sociales y Tecnologías de la Información y la Comunicación en Educación: aprendizaje colaborativo, diferencias de género, edad y preferencias. RED. Revista de Educación a Distancia, 51(1), 1-23. https://bit.ly/3aQVP5U

Calle, M., Saavedra, L., Velásquez, V. (2016). Mediaciones pedagógicas para el desarrollo de las inteligencias múltiples en Educación Superior. Revista Pertinencia Académica, Vol. 1, 37-52. https://bit.ly/3pLi4OT

Chancusig, J., Guilcaso, J., Montaluisa, R., Soria, M. (2019). El aprendizaje y las tecnologías de información y comunicación en la educación superior en Ecuador. Revista Científica Mundo de la Investigación y el Conocimiento, 3(1), 1340-1352. https://bit.ly/38J1kAB

Díaz, C. (2009). Las competencias TIC y la integración de las tecnologías de información y comunicación de los docentes de la universidad católica del Maule. (Tesis de Maestría en Educación). Universidad de Chile, Santiago de Chile - Chile. https://bit.ly/3pkZLQj

García, G. (2014). Inteligencias múltiples y variables psicoeducativas en estudiantes de secundaria. (Tesis Doctoral). Universidad de Alicante, Alicante - España. https://bit.ly/2Jj2zhu

Gardner, H. (2015). Inteligencias múltiples: La teoría en la práctica. Barcelona: Paidós Educación.

Hermosa, P. (2015). Influencia de las tecnologías de información y comunicación (TIC) en el proceso enseñanza-aprendizaje: una mejora de las competencias digitales. Rev. Cient. Gen. José María Córdova, 13(16), 121-132. https://bit.ly/3htqkA8

Hernández, R., Mendoza, C. (2018). Metodología de la investigación: las rutas cuantitativa, cualitativa y mixta. Ciudad de México: Mc Graw Hill. 
Hernández, R., Fernández, C., Baptista, P. (2014). Metodología de la investigación (Sexta ed.). México: McGraw-Hill.

Hidalgo, S., Sospedra, M., Martínez, I. (2018). Análisis de las inteligencias múltiples y creatividad en universitarios. Ciencias Psicológicas, 12(2), 271-280. http://dx.doi.org/10.22235/cp.v12i2.1691

Islas, C. (2017). La implicación de las TIC en la educación: Alcances, Limitaciones y Prospectiva. RIDE. Rev. Iberoam. Investig. Desarro. Educ, 15(8), 861-876. https://bit.ly/3mI9hLw

Matheus, S. (2011). Inteligencia para todos. Lima: Perú Publicación.

Matute, V. (2013). Uso de las tecnologías de información y comunicación en las clases de inglés en las instituciones públicas de educación secundaria del casco urbano de la ciudad de Santa Bárbara. (Tesis de Maestría en Educación). Universidad Pedagógica Nacional Francisco Morazán, San Pedro Sula - Honduras. https://bit.ly/3mKfbvD

Mesa, C. (2018). Caracterización de las inteligencias múltiples de estudiantes de 2 do año de la carrera de Medicina. Revista Médica Electrónica, 40(2), 298-310. https://bit.ly/3pyvkGl

Montilla, A., Agüero, E., Mora, L. (2018). Geotecnologías en la enseñanza de geociencias en el instituto pedagógico de Maturín. Revista Rehuso, 3(3), 1-12. https://bit.ly/34ROEpZ

Moreno, J., Planells, B. (2016). Propuesta para la implementación de la teoría de las Inteligencias Múltiples en el sistema de Educación Infantil en España. Revista Electrónica Interuniversitaria de Formación del Profesorado, 3(19), 199-207. https://www.redalyc.org/pdf/2170/217047011018.pdf

Ñaupas, H., Palacios, J., Valdivia, M., Romero, H. (2018). Metodología de la investigación Cuantitativa-Cualitativa y redacción de la tesis. Colombia: DGP Editores.

UNESCO. (2019). Las TIC en la educación. https://bit.ly/3nJs36D

UNESCO. (2019b). ¿Qué hace la UNESCO en relación con el uso de las TIC en la educación? https://bit.ly/2JdPqWX

Palella, S., Martins, F. (2012). Metodología de la investigación cuantitativa. Caracas, Venezuela: Fedupel.

Paucar, L., Borja, Y., Salazar, A., Chancusig, J., Bedón, P. (2017). Estrategias de planificación del área informática para potencializar el uso de las tecnologías de información y comunicación en Instituciones de Educación Superior (Universidad Técnica de Cotopaxi). Revista Arjé, 11(21), 233-244. https://bit.ly/37THpzO

Pérez, M. (2017). Uso actual de las tecnologías de información y comunicación en la educación médica. Revista Médica Herediana, 28(4), 258-265. https://bit.ly/3rBqwlu

Pérez, R., Mercado, P., Martínez, M., Mena, E., Partida, J. (2018). La sociedad del conocimiento y la sociedad de la información como la piedra angular en la innovación tecnológica educativa. RIDE. Rev. Iberoam. Investig. Desarro. Educ, 847-870. https://bit.ly/38IhQkH

Rosas, J., Zúñiga, P. (2010). Estadística Descriptiva E Inferencial I. Fascículo 3. Correlación y regresiones lineales. Colegio de Bachilleres. 